OPEN ACCESS

Edited by:

Li Chen,

The Chinese Academy of Sciences,

China

Reviewed by:

Xin-Cheng Zhao,

Henan Agricultural University, China

Wei Xu,

Commonwealth Scientific and Industrial Research Organisation,

Australia

*Correspondence:

William B. Walker III

william.b.walker.ii@s/u.se

Specialty section:

This article was submitted to

Chemical Ecology,

a section of the journal

Frontiers in Ecology and Evolution

Received: 26 February 2016

Accepted: 29 March 2016

Published: 19 April 2016

Citation:

Walker WB III, Jacquin-Joly E and Hill

SR (2016) Editorial: Functional

Characterization of Insect

Chemoreceptors: Receptivity Range and Expression.

Front. Ecol. Evol. 4:37.

doi: 10.3389/fevo.2016.00037

\section{Editorial: Functional Characterization of Insect Chemoreceptors: Receptivity Range and Expression}

\author{
William B. Walker III ${ }^{1 *}$ Emmanuelle Jacquin-Joly ${ }^{2}$ and Sharon R. Hill ${ }^{1}$ \\ ${ }^{1}$ Unit of Chemical Ecology, Department of Plant Protection Biology, Swedish University of Agricultural Sciences, Alnarp, \\ Sweden, ${ }^{2}$ Institut National de la Recherche Agronomique, Institute of Ecology and Environmental Sciences of Paris, \\ Versailles, France
}

Keywords: chemical ecology, molecular biology, insects, chemosensory, olfaction, chemoreceptors, gustation, gene expression

The Editorial on the Research Topic

Functional Characterization of Insect Chemoreceptors: Receptivity Range, Expression, and Evolution

Chemosensory systems play an oversize role in shaping the life of an insect, such that fundamental behaviors - mating, food choice and seeking, predator and parasitoid avoidance, and egg-laying-are strongly regulated by external chemical stimuli. The recent focus on the molecular mechanisms of chemosensory detection in insect chemical ecology research has identified canonical chemosensory receptors in insects that consist of odorant receptors (ORs), gustatory receptors (GRs), and ionotropic receptors (IRs). Much has been learned about the structure, function and evolution of chemosensory receptors since the initial discovery of ORs in Drosophila melanogaster in 1999, however, many outstanding questions remain. With this research topic, we aim to shine a light on expression patterns, reception properties, and evolutionary trends pertinent to insect chemosensory receptors. While intended to cover all chemosensory receptor families, this research topic is clearly biased toward ORs, reflecting the paucity of research conducted on GRs and IRs.

\section{ECOLOGICAL AND BEHAVIORAL RELEVANCE OF CHEMORECEPTORS}

The detection of ecologically relevant cues via chemoreceptors ultimately induces behavioral changes. The review by Depetris-Chauvin et al. provides an up-to-date look at chemical communication in flies and fitness-related behaviors, including courtship. The importance of both internal and external context for the interpretation of chemical cues is highlighted throughout the lifecycle of the fly. The authors suggest that plasticity in chemoreceptive behavior may be a result of chemoreceptor repertoire modulation, reflecting the distinct physiological requirements of various ecological environments inhabited at different life stages.

Modulation of chemosensory-based behaviors is a dynamic process that occurs subsequent to the processing of sensory stimuli, but the molecular mechanisms underlying such changes are not yet known. One supported hypothesis points toward a role for modulation of chemosensory gene expression in generating changes in behavior. Latorre-Estivalis et al. provide support for this 
hypothesis, demonstrating the effects of blood-feeding and development on expression levels of OR and IR co-receptors in the important Chagas disease vector, Rhodnius prolixus.

\section{EVOLUTION OF RECEPTOR TUNING PARADIGMS}

Peripheral coding of signals contributes to the interpretation of chemosensory information in the insect nervous system. The hypothesis of peripheral combinatorial coding of chemical stimuli is contrasted to the labeled-line hypothesis. Re-examining these principles at the molecular level brings into play the concept of generalist vs. specialist receptors, with broader and more narrow receptor tuning ranges, respectively. Bohbot and Pitts explore these themes for insect ORs, and propose a general prevalence of specialized receptors, while acknowledging that pharmacological receptivity ranges of receptors may be broader. Exploring this concept further, Andersson et al. examine the principles of OR tuning in evolutionary contexts. Examples are provided for both broad and narrow tuning, and scenarios are presented wherein evolutionary conditions would favor tilting toward either model.

A central dogma concerning insect olfactory information flow is that one olfactory sensory neuron (OSN) expresses one OR subtype, and axons of OSNs expressing the same OR converge within the same glomerular cluster in the primary olfactory processing center of the brain, the antennal lobe. However, there are exceptions to these rules and Karner et al. showcase this, reporting the co-expression of four to six genomically clustered OR genes in the same OSN in a mosquito. These OSNs may thus serve as broadly tuned sensors.

Above and beyond investigations into model organisms with sequenced genomes, the advent of high-throughput transcriptomic sequencing (RNA-Seq) has led to a dramatic increase in the breadth of gene identification and characterization. Here, the application of RNA-Seq methodology is highlighted with a description of chemosensory gene families, including ORs and IRs, in the Colorado potato beetle (Liu et al.). The identification of beetle ORs with sexually biased expression patterns suggests a molecular basis for known sexually dimorphic olfactory-based behaviors.

A logical step following chemosensory receptor discovery is functional characterization. Receptor deorphanization is defined by the process of identifying key activating ligands for chemosensory receptors and describing their receptive range; a difficult task for non-model insects. Using the in vivo deorphanization systems that have been developed in D. melanogaster for transgenic expression and functional characterization of insect ORs, Gonzalez et al. provide detailed step-by-step protocols to facilitate widespread accessibility and adoption of this methodology.

Three reports in this Research Topic utilize fly transgenic systems to characterize ORs, (Gonzalez et al.) and pheromone receptors (PRs) (de Fouchier et al.; Bengtsson et al.) in moths, each making distinct contributions to the fundamental knowledge that underlies the molecular mechanisms of olfactory detection. Gonzalez et al. report that homologous ORs from two distantly related moth species respond similarly to the same set of odorant ligands. These data support functional conservation in homologous ORs and provide hypotheses concerning the interconnection of structure and function with respect to modeling odorant ligand interactions with critical amino acid residues in the receptor proteins.

While many hypotheses exist, there is still a prominent gap in the knowledge concerning the mechanism(s) underlying chemoreceptors' specific interaction with their chemical ligands. Almeida et al. provide an important contribution toward the theoretical framework of this knowledge with their examination of site-specific evolutionary rates in GRs and IRs in a non-insect lineage. Relaxed selective constraints are a prominent feature of duplicated genes, permitting neo-functionalization of redundant gene models. Furthermore, rapid evolution of specific amino acid residues is biased toward extracellular domains, which are predicted to be involved in ligand binding.

\section{MOTH PHEROMONE RECEPTORS}

The chemical ecology of moth pre-mating communication has been widely studied, from pheromone component identification and biosynthesis to PR characterization. This facet of insect chemical ecology has persisted in the spotlight largely due to the prominence of moths as agricultural pests, as well as the successfully demonstrated potential for hacking the olfactory system as a means of species specific biorational pest control. Accordingly, Zhang and Löfstedt provide a thorough review of state of the art knowledge on moth PRs with respect to sequence, function and evolution in the context of their pheromone ligands.

Exploring the underpinnings of moth mating systems, de Fouchier et al. report on two PRs that respond to similar, but not completely overlapping, sets of minor pheromone components. This report places these receptors in an evolutionary context, evaluating their position within broader lineages of moth PRs, as well as examining differential evolutionary pressures on specific amino acid residues. The latter point reiterates a need for a greater understanding of the mechanism(s) by which chemoreceptors interact with their ligands.

Continuing with the theme on evolution of PRs, Bengtsson et al. describe a codling moth OR that responds to a host plant volatile, pear ester, but clusters phylogenetically with the welldescribed sub-family clade of moth PRs. Its response to a host plant volatile was, at first glance, surprising, but the receptor displays hallmark features of PRs, namely, high specificity and sensitivity to its key ligand.

Evolution of pheromone communication requires the coadaptation of pheromone and receptor, suggesting a degree of variation in the sequence and expression of each within a population. Alternative splicing represents one cellular mechanism whereby an increased diversity of protein products can stem from a relatively limited number of genes, providing functional plasticity in chemoreception. Here, Garczynski and Leal provide the first known report on splicing of the 3'/Cterminal region of $\mathrm{PR}$ sequence. The functional implications 
of this remain unknown, and further research is required on structure function relationships and ligand binding properties of alternatively spliced receptors.

\section{CONCLUDING REMARKS}

We are very grateful to all authors who contributed articles to this Topic, illustrating most of the facets of studies currently conducted on insect chemoreceptors. We also thank all reviewers and affiliated scientific editors who helped us in reaching the highest quality standards, as well as the Frontiers editorial team for invaluable and consistent support and encouragement.

\section{AUTHOR CONTRIBUTIONS}

WW, $\mathrm{SH}$, and EJJ wrote, edited, and finalized this document.

\section{FUNDING}

This report was supported by the Linnaeus initiative "Insect Chemical Ecology, Ethology, and Evolution (IC-E3)" [Formas, SLU], Carl Tryggers Stiftelse för Vetenskaplig Forskning [Sweden], and Agence Nationale de la Recherche [ANR-09BLAN-0239-01, France].

Conflict of Interest Statement: The authors declare that the research was conducted in the absence of any commercial or financial relationships that could be construed as a potential conflict of interest.

Copyright (c) 2016 Walker, Jacquin-Joly and Hill. This is an open-access article distributed under the terms of the Creative Commons Attribution License (CC BY). The use, distribution or reproduction in other forums is permitted, provided the original author(s) or licensor are credited and that the original publication in this journal is cited, in accordance with accepted academic practice. No use, distribution or reproduction is permitted which does not comply with these terms. 